\title{
Effective Mass Generation of Off-diagonal Gluons and Abelian Dominance in the Maximally Abelian Gauge in QCD
}

\author{
K. Amemiya and H. Suganuma ${ }^{a}$ \\ ${ }^{a}$ Research Center for Nuclear Physics (RCNP), Osaka University, Mihogaoka 10-1, Ibaraki, Osaka \\ 567-0047, Japan
}

We study the properties of gluons in QCD in the maximally abelian (MA) gauge. In the MA gauge, the off-diagonal gluon behaves as the massive vector boson with the mass $M_{\text {off }} \simeq 1.2 \mathrm{GeV}$, and therefore the off-diagonal gluon cannot carry the long-range interaction for $r \gg M_{\text {off }}^{-1} \simeq 0.2$ fm. The essence of the infrared abelian dominance in the MA gauge is physically explained with the generation of the offdiagonal gluon mass $M_{\text {off }} \simeq 1.2 \mathrm{GeV}$ induced by the MA gauge fixing, and the off-diagonal gluon mass generation would predict general infrared abelian dominance in QCD in the MA gauge. We report also the off-diagonal gluon propagator at finite temperature.

\section{Introduction}

The quark-confinement mechanism is one of the most important subjects in the nonperturbative QCD. In the dual-superconductor picture [1], the quark-confinement mechanism can be physically interpreted with the dual Meissner effect. In this context, the MA gauge has been paid much attention to construct the dual-superconductor picture from QCD 2 2 4. In the MA gauge, diagonal gluon components behave as neutral gauge fields like photons, and off-diagonal gluon components behave as charged matter fields on the residual abelian gauge symmetry. In the MA gauge, the diagonal part of the gluon field has been demonstrated to play a dominant role to the nonperturbative quantities like confinement [5] and chiral symmetry breaking [6]. On the other hand, the off-diagonal part of the gluon field does not contribute to the long-range phenomena. This, abelian dominance [3], is one of the key concepts to link the dual-superconductor picture from QCD.

\section{Mass-Generation Hypothesis on Off- diagonal Gluons in the MA gauge}

Abelian dominance for the infrared region, which we call "infrared abelian dominance", is usually discussed on the role of the diagonal component of the gluon field. However, in terms of the off-diagonal gluon, infrared abelian dominance can be expressed that off-diagonal gluon components are inactive at the infrared scale of QCD and can be neglected for the argument of the nonperturbative QCD. As a possible physical interpretation for infrared abelian dominance as infrared inactivity of off-diagonal gluon, we conjecture that the effective mass of the off-diagonal gluon $A_{\mu}^{ \pm} \equiv \frac{1}{\sqrt{2}}\left(A_{\mu}^{1} \pm i A_{\mu}^{2}\right)$ is induced in the MA gauge. If $A_{\mu}^{ \pm}$acquires a large effective mass $M_{\text {off }}$, the off-diagonal gluon propagation is limited within the short range as $r \lesssim M_{\text {off }}^{-1}$. This mass-generation hypothesis on off-diagonal gluon in the MA gauge is formally expressed in QCD as follows [5, 7,8]. The SU(2) QCD partition functional in the MA gauge is expressed as

$Z_{\mathrm{QCD}}^{\mathrm{MA}}=\int D A_{\mu} e^{i S_{\mathrm{QCD}}\left[A_{\mu}\right]} \delta\left(\Phi_{\mathrm{MA}}^{ \pm}\left[A_{\mu}\right]\right) \Delta_{\mathrm{PF}}\left[A_{\mu}\right]$,

where $\Delta_{\mathrm{FP}}\left[A_{\mu}\right]$ denotes the Faddeev-Popov determinant. Here, $\Phi_{\mathrm{MA}}^{ \pm}\left[A_{\mu}\right]$ denotes the offdiagonal component of $\Phi_{\mathrm{MA}}\left[A_{\mu}\right] \equiv\left[\hat{D}_{\mu},\left[\hat{D}^{\mu}, \tau^{3}\right]\right]$ which is diagonalized in the MA gauge [5.,8,9], and therefore the MA gauge fixing is provided by $\delta\left(\Phi_{\mathrm{MA}}^{ \pm}\left[A_{\mu}\right]\right)$. The mass-generation hypothesis of off-diagonal gluon $A_{\mu}^{ \pm}$is expressed as

$Z_{\mathrm{QCD}}^{\mathrm{MA}}=\int \mathcal{D} A_{\mu}^{3} e^{i S_{\mathrm{Abel}}\left[A_{\mu}^{3}\right]} \int \mathcal{D} A_{\mu}^{ \pm} e^{i S_{\mathrm{off}}^{M}\left[A_{\mu}^{ \pm}\right]} \mathcal{F}\left[A_{\mu}\right]$.

Here, $S_{\text {off }}^{M}\left[A_{\mu}^{ \pm}\right]$denotes the $\mathrm{U}(1)_{3}$-invariant action of the off-diagonal gluon with the effective mass 
$M_{\text {off }}$ as

$$
\begin{aligned}
S_{\mathrm{off}}^{M}\left[A_{\mu}^{ \pm}\right] \equiv \int d^{4} x\left\{-\frac{1}{2}\left(D_{\mu}^{\mathrm{Abel}} A_{\nu}^{+}-D_{\nu}^{\mathrm{Abel}} A_{\mu}^{+}\right)\right. \\
\left.\times\left(D_{\mathrm{Abel}}^{\mu *} A_{-}^{\nu}-D_{\mathrm{Abel}}^{\nu *} A_{-}^{\mu}\right)+M_{\mathrm{off}}^{2} A_{\mu}^{+} A_{-}^{\mu}\right\}
\end{aligned}
$$

with the $\mathrm{U}(1)_{3}$ covariant derivative $D_{\mu}^{\mathrm{Abel}} \equiv \partial_{\mu}+$ $i e A_{\mu}^{3}$. Here, $S_{\text {Abel }}\left[A_{\mu}^{3}\right]$ is the effective action of the diagonal gluon component, and $\mathcal{F}\left[A_{\mu}\right]$ is a $\mathrm{U}(1)_{3}$-invariant smooth functional in comparison with $\exp \left\{i S_{\text {off }}^{M}\left[A_{\mu}^{ \pm}\right]\right\}$at least in the infrared region. Since the effective mass $M_{\text {off }}$ is closely related to the off-diagonal gluon propagation, we study the gluon propagator in the MA gauge in terms of the interaction range using the $\mathrm{SU}(2)$ lattice QCD simulations [5, 7,8 .

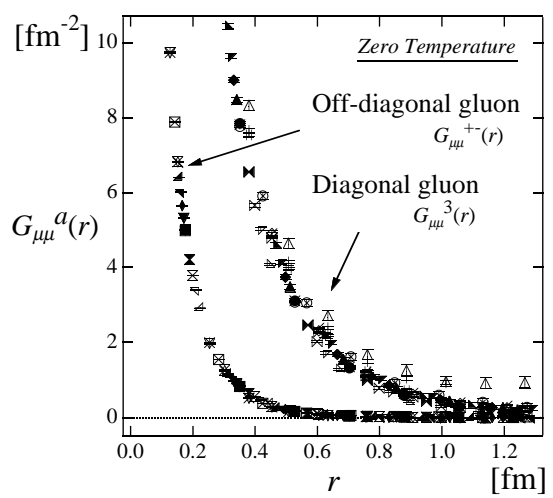

Figure 1. The scalar-type gluon propagator $G_{\mu \mu}^{a}(r)$ as the function of the 4-dimensional distance $r$ in the MA gauge with $2.2 \leq \beta \leq 2.4$ and various lattice size $\left(12^{3} \times 24,16^{4}, 20^{4}\right)$.

\section{Effective Mass of the Off-diagonal Gluon in the MA Gauge}

In the Euclidean QCD, the MA gauge is defined by minimizing $R_{\text {off }}\left[A_{\mu}\right] \equiv e^{2} \int d^{4} x A_{\mu}^{+}(x) A_{-}^{\mu}(x)$. In the MA gauge, the off-diagonal gluon components are forced to be as small as possible by the $\mathrm{SU}(2)$ gauge transformation [5]. In the MA gauge, the $\mathrm{SU}(2)$ gauge symmetry is reduced into the $\mathrm{U}(1)_{3}$ gauge symmetry. As for the residual $\mathrm{U}(1)_{3}$ gauge symmetry, we impose the $\mathrm{U}(1)_{3}$ Landau gauge fixing to extract most continuous

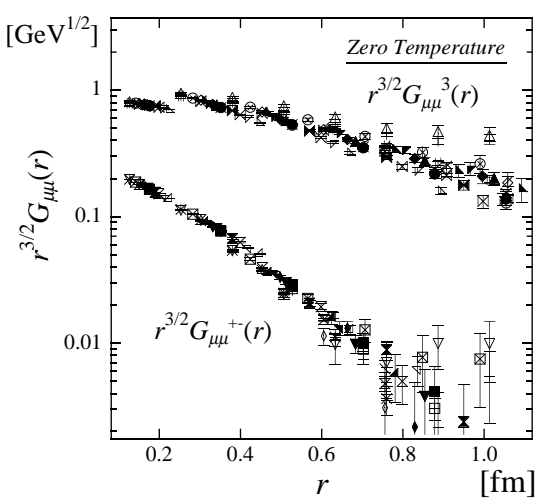

Figure 2. The logarithmic plot for the scalar correlation $r^{3 / 2} G_{\mu \mu}^{a}(r)$. The off-diagonal gluon propagator behaves as the Yukawa-type function, $G_{\mu \mu}^{+-} \sim \frac{\exp \left(-M_{\text {off }} r\right)}{r^{3 / 2}}$.

gauge configuration under the MA gauge constraint and to compare with the continuum theory [5, 7,8$]$.

To investigate the off-diagonal gluon mass $M_{\text {off }}$, we study the Euclidean gluon propagator $G_{\mu \nu}^{a b}(x-y) \equiv\left\langle A_{\mu}^{a}(x) A_{\nu}^{b}(y)\right\rangle$ in the MA gauge with the $\mathrm{U}(1)_{3}$ Landau gauge fixing [5,7,8]. The continuum gluon field $A_{\mu}^{a}(x)$ is extracted from the link variable as $U_{\mu}(s)=\exp \left(\right.$ iae $\left.A_{\mu}^{a}(s) \frac{\tau^{a}}{2}\right)$. Here, the scalar-type gluon propagator $G_{\mu \mu}^{a}(r) \equiv$ $\sum_{\mu=1}^{4}\left\langle A_{\mu}^{a}(x) A_{\mu}^{a}(y)\right\rangle$ is useful to observe the interaction range of the gluon, because it depends only on the four-dimensional Euclidean distance $r \equiv \sqrt{\left(x_{\mu}-y_{\mu}\right)^{2}}$.

We show in Fig.1 $G_{\mu \mu}^{3}(r)$ and $G_{\mu \mu}^{+-}(r) \equiv$ $\sum_{\mu=1}^{4}\left\langle A_{\mu}^{+}(x) A_{\mu}^{-}(y)\right\rangle=\frac{1}{2}\left\{G_{\mu \mu}^{1}(r)+G_{\mu \mu}^{2}(r)\right\}$ in the MA gauge with the $\mathrm{U}(1)_{3}$ Landau gauge fixing. In the MA gauge, $G_{\mu \mu}^{3}(r)$ and $G_{\mu \mu}^{+-}(r)$ manifestly differ. The diagonal-gluon propagator $G_{\mu \mu}^{3}(r)$ takes a large value even at the long distance. In fact, the diagonal gluon $A_{\mu}^{3}$ in the MA gauge propagates over the long distance. On the other hand, the off-diagonal gluon propagator $G_{\mu \mu}^{+-}(r)$ rapidly decreases and is negligible for $r \gtrsim 0.4 \mathrm{fm}$ in comparison with $G_{\mu \mu}^{3}(r)$. Then, the off-diagonal gluon $A_{\mu}^{ \pm}$seems to propagate only within the short range as $r \lesssim 0.4 \mathrm{fm}$. Thus, infrared abelian dominance for the gluon propagator is found in the MA gauge. Since the massive vector-boson propagator with the 
mass $M$ takes a Yukawa-type asymptotic form as $G_{\mu \mu}(r) \sim \frac{M^{1 / 2}}{r^{3 / 2}} \exp (-M r)$ for $M r>1$, the effective mass $M_{\text {off }}$ of the off-diagonal gluon $A_{\mu}^{ \pm}(x)$ can be evaluated from the slope of the logarithmic plot of $r^{3 / 2} G_{\mu \mu}^{+-}(r) \sim \exp \left(-M_{\mathrm{off}} r\right)$ as shown in Fig.2. The off-diagonal gluon $A_{\mu}^{ \pm}(x)$ behaves as the massive vector boson with $M_{\text {off }} \simeq 1.2 \mathrm{GeV}$

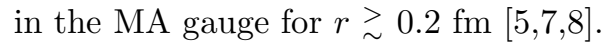

\section{Gluon Propagator in the MA Gauge at Finite Temperature}

We study also the scalar-type gluon propagator in the MA gauge at finite temperature 10. We measure the spatial correlation of $G_{\mu \mu}^{a}(R, 0) \equiv \sum_{\mu=1}^{4}\left\langle A_{\mu}^{a}(\vec{x}, 0) A_{\mu}^{a}(\vec{y}, 0)\right\rangle$ in the MA gauge with the $\mathrm{U}(1)_{3}$ Landau gauge fixing. Here, $G_{\mu \mu}^{a}(R, 0)$ depends on the threedimensional distance $R \equiv|\vec{x}-\vec{y}|$ at finite temperature. In Fig. 3, we show the preliminary results of the numerical simulation for $G_{\mu \mu}^{3}(R, 0)$ and $G_{\mu \mu}^{+-}(R, 0) \equiv \sum_{\mu=1}^{4}\left\langle A_{\mu}^{+}(\vec{x}, 0) A_{\mu}^{-}(\vec{y}, 0)\right\rangle=$ $\frac{1}{2}\left\{G_{\mu \mu}^{1}(R, 0)+G_{\mu \mu}^{2}(R, 0)\right\}$ in the MA gauge. Even at finite temperatures, we find that abelian dominance holds for the spatial propagation of gluons in the region of $R \gtrsim 0.4 \mathrm{fm}$. In Fig. 3, $G_{\mu \mu}^{3}(R, 0)$ largely changes between the confinement and the deconfinement phases. On the other hand, $G_{\mu \mu}^{+-}(R, 0)$ is almost the same even in the deconfinement phase. The off-diagonal gluon $A_{\mu}^{ \pm}$ in the MA gauge seems to propagate within the short-range region as $R \lesssim 0.4 \mathrm{fm}$ almost irrespective of the medium of QCD.

\section{Summary and Concluding Remarks}

We have studied the gluon propagator in the MA gauge in terms of the interaction range. We have found that infrared abelian dominance for the gluon propagator in the MA gauge, and the interaction range of the off-diagonal gluon is restricted within a short distance. The off-diagonal gluon behaves as the massive vector boson with the effective mass $M_{\text {off }} \simeq 1.2 \mathrm{GeV}$. Therefore, the off-diagonal gluon $A_{\mu}^{ \pm}$can propagate only within the short range as $r \underset{\sim}{\sim} M_{\text {off }}^{-1} \simeq 0.2 \mathrm{fm}$, and cannot contribute to the infrared QCD physics in the MA gauge. Then, abelian dominance holds

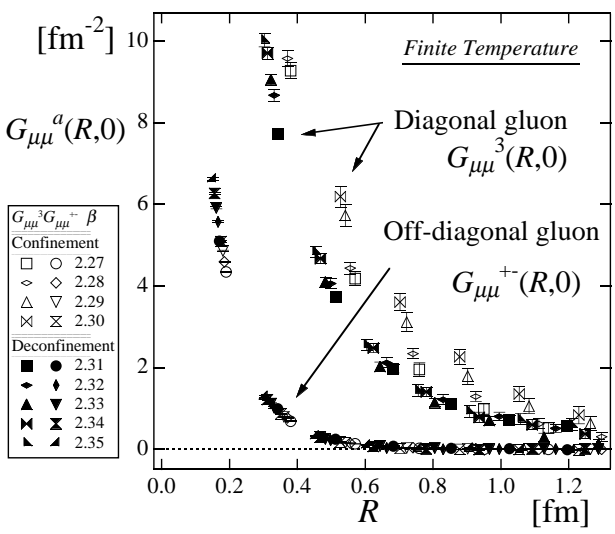

Figure 3. The scalar-type gluon spatial correlation $G_{\mu \mu}^{a}(R, 0)$ at finite temperatures as the function of the 3-dimensional distance $R$ in the MA gauge with $2.27 \leq \beta \leq 2.35$ on $12^{2} \times 4 \times 24$ lattice.

for the long-distance physics with $r \gg M_{\text {off }}^{-1}$ in QCD in the MA gauge and $M_{\text {off }}^{-1} \simeq 0.2 \mathrm{fm}$ can be regarded as the critical scale of abelian dominance. In this way, essence of infrared abelian dominance in the MA gauge can be physically interpreted with the effective off-diagonal gluon mass $M_{\text {off }}$ induced by the MA gauge fixing, and the off-diagonal gluon mass generation seems to predict the general infrared abelian dominance in QCD in the MA gauge.

\section{REFERENCES}

1. Y. Nambu, Phys. Rev. D10 (1974) 4262.

2. G. 't Hooft, Nucl. Phys. B190 (1981) 455.

3. Z. F. Ezawa and A. Iwazaki, Phys. Rev. D25 (1982) 2681; D26 (1982) 631.

4. H. Suganuma, S. Sasaki and H. Toki, Nucl. Phys. B435 (1995) 207 and its references.

5. H. Suganuma, H. Ichie, A. Tanaka and K. Amemiya, PTP Suppl. 131 (1998) 559.

6. O. Miyamura, Phys. Lett. B353 (1995) 91.

7. K. Amemiya and H. Suganuma, Int. Symp. on Innovative Computational Methods in Nuclear Many-Body Problems (World Scientific, 1998) p.284: hep-lat/9712028.

8. K. Amemiya, H. Suganuma, hep-lat/9811035.

9. H.Ichie and H.Suganuma, Nucl. Phys. B548 (1999) 365; Phys. Rev. D60 (1999) 077501. 
10. K. Amemiya, H. Suganuma, in preparation. 